\title{
La Ley de Modernización de la Inocuidad de Alimentos de la FDA: Énfasis en la Prevención para la Industria de Alimentos Salvadoreña.
}

\author{
The FDA Food Safety Modernization Act: \\ Emphasis on prevention for Salvadoran food industry.
}

Alicia Urquilla de Castaneda

Investigadora Asociada, UFG 



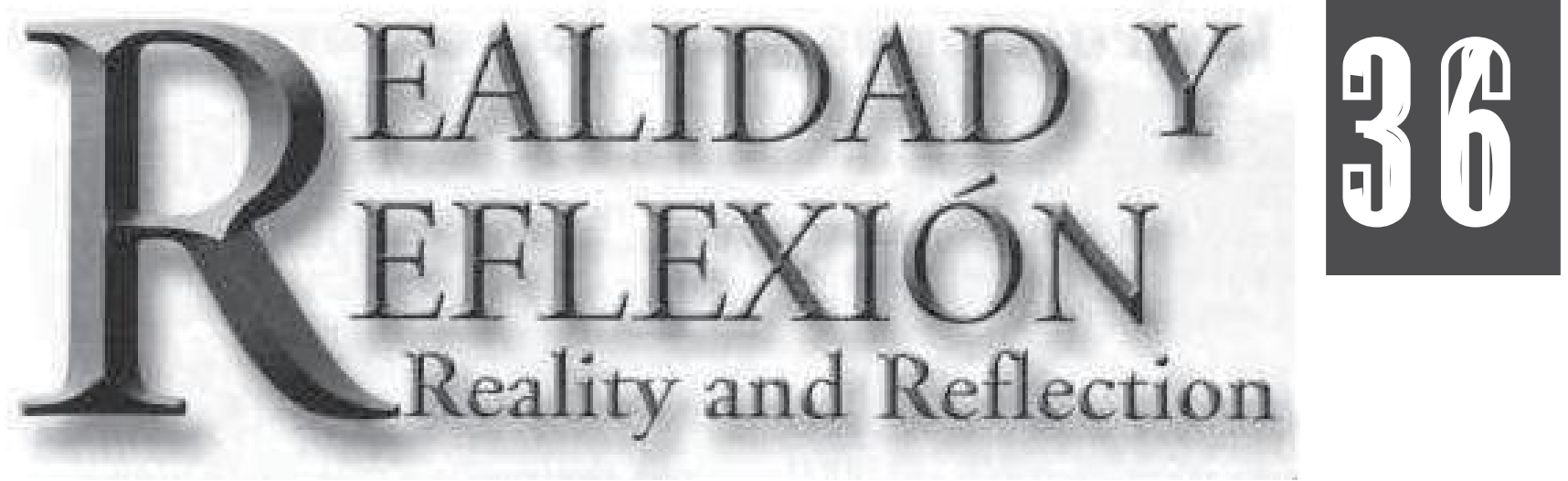

Año 12, No. 36 San Salvador, El Salvador, Centroamérica Year 12, Nr. 36
San Salvador, EI Salvador, Central America
Revista Cuatrimestral

Quarterly J ournal
Septiembre-Diciembre 2012

September-December 2012

\section{La Ley de Modernización de la Inocuidad de Alimentos de la FDA: Énfasis en la Prevención para la Industria de Alimentos Salvadoreña. The FDA Food Safety Modernization Act: Emphasis on prevention for Salvadoran food industry.}

Alicia Urquilla de Castaneda Investigadora Asociada UFG

\section{Resumen}

Este artículo se refiere a la propuesta de Ley que la FDA ha realizado a través de FSMA (Food Safety Modernization Act), en la cual se pretende brindar los últimos cambios respecto a esta Ley, con el objetivo de implementar acciones y fortalecer el conocimiento de la industria salvadoreña que exporta sus productos al mercado de los Estados Unidos. Asimismo, ofrecer los resultados sobre los rechazos que sufren los productos de las empresas salvadoreñas al llegar a las aduanas de los EEUU, para los meses de noviembre y diciembre del año 2012, detectar cuáles son las causas de estas detenciones en el periodo mencionado y de esta manera ir monitoreando en qué áreas se necesita apoyar a la industria de alimentos y bebidas de El Salvador por parte del sector académico y ocasionar, que tanto el sector público como el privado puedan generar propuestas de acción para este fin. Descriptores: TECNOLOGÍA DE ALIMENTOS, CONSERVACIÓN DE ALIMENTOS, HIGIENE DE LOS ALIMENTOS, CONTROL DE ALIM ENTOS, AGENCIA DE ALIM ENTOS Y M EDICAM ENTOS (FDA), ,

\section{Summary}

This article refers to the proposed law that FDA has made through FSMA (Food Safety Modernization Act), which aims to provide the latest updates regarding this Act, in order to implement actions and strengthen the knowledge of Salvadoran industry exports its products to the U.S. market. Also provide results on rejections experienced by Salvadoran firms products arriving at U.S. customs, for the months of November and December 2012, identify the causes of these arrests in the aforementioned period and this way going monitoring which areas needed to sup port the food and beverage industry in El Salvador by the academic sector and result, both the public and private sectors to generate proposals for action for this purpose. Descriptors: TECHNOLOGY OF FOOD, FOOD STORE, FOOD HYGIENE, CONTROL OF FOOD, FOOD AND DRUG AGENCY (FDA), 


\section{Introducción}

\section{Antecedentes sobre la propuesta de Ley de Modernización de la Inocuidad de los Alimentos (FSMA) ${ }^{1}$ de la FDA.}

"Alrededor de 48 millones de personas (1 de cada 6 estadounidenses) se enferman, 128,000 son hospitalizadas y 3,000 mueren cada año de enfermedades transmitidas por los alimentos, según datos recientes de los Centros para el Control y la Prevención de Enfermedades. Esto es una carga considerable para la salud pública de los EEUU que en gran parte puede prevenirse".

El 16 de Enero del 2013 la Administración de Alimentos y Medicamentos de los Estados Unidos de América (FDA por sus siglas en inglés) propuso dos nuevas normas de inocuidad de alimentos que ayudarán a prevenir las enfermedades transmitidas por alimentos (ETA) en los Estados Unidos.

Las normas propuestas implementan la Ley de Modernización de la Inocuidad de Alimentos, una ley basada en el sentido común que cambia el enfoque reactivo de la inocuidad de alimentos hacia uno preventivo. La prevención es una responsabilidad compartida entre todos los participantes del sistema alimentario.

La primera norma, sobre controles preventivos de alimentos para humanos, va a requerir que los productores de alimentos, los cuales serán vendidos en los Estados Unidos, ya sean elaborados en instalaciones locales 0 en el extranjero, desarrollen un plan formal para prevenir que sus productos alimenticios causen alguna ETA.

El plan escrito se establecerá con el objetivo de identificar los peligros potenciales a la inocuidad de los alimentos, estableciendo los pasos a seguir para manejarlos, verificando que los pasos definidos funcionen, y describir cómo corregir cualquier problema que surja.

La FDA está proponiendo que muchos productores de alimentos cumplan con esta nueva norma de controles preventivos un año después que las normas finales sean publicadas en el Federal Register. A empresas pequeñas y muy pequeñas se les dará tiempo adicional para su cumplimiento.

La segunda norma propone la exigencia de estándares de inocuidad en la producción y cosecha de frutas y vegetales en fincas. La regla aplica tanto a productos locales como a los importados.

Se va a requerir que las fincas que cultivan, cosechan, empacan o mantienen frutas y vegetales cubiertas por esta norma propuesta, sigan ciertos estándares dirigidos a prevenir la contaminación de sus productos.

La FDA está proponiendo que las grandes fincas cumplan con la mayoría de los requisitos de inocuidad de frutas y vegetales 26 meses después que la norma final sea publicada en el Federal Register. Empresas pequeñas y muy pequeñas tendrán tiempo adicional para su cumplimiento, y todas las fincas 
tendrán tiempo adicional para cumplir con ciertos requisitos relacionados a la calidad del agua.

Las enfermedades transmitidas por los alimentos se pueden prevenir si todos los actores que conforman la cadena productiva alimenticia actúan responsablemente en cada etapa que controla los peligros que pueden provocar una enfermedad. Bajo la nueva Ley, la FDA tendrá nuevas herramientas enfocadas en la prevención y un marco regulatorio claro para ayudar a hacer mejoras sustanciales en nuestro enfoque hacia la inocuidad de los alimentos. La nueva Ley también mejora significativamente la facultad de la FDA en lograr mayor control sobre los millones de productos alimenticios que llegan anualmente a los EEUU desde otros países.

\section{La Nueva Propuesta de Ley}

La Ley de Modernización de la Inocuidad de los Alimentos (Food Safety Modernization Act -FSMA) de la FDA, permite a la FDA proteger mejor la salud pública al garantizar la seguridad del suministro de alimentos. La ley también otorga a la FDA nuevas facultades de ejecución destinadas a lograr índices de cumplimiento más altos con normas de inocuidad de los alimentos basadas en la prevención y los riesgos, y a responder mejor a los problemas, y contenerlos, cuando estos ocurren. La ley también brinda a la FDA nuevos instrumentos importantes para someter a los alimentos importados a las mismas normas que los alimentos nacionales (EEUU) y le ordena establecer un sistema integrado nacional de inocuidad de los alimentos en asociación con las autoridades estatales y locales.

A continuación se mencionan algunos de los nuevos mandatos y facultades claves de la FDA. Las fechas de aplicación específicas estipuladas en la ley se indican en paréntesis:

\section{Prevención}

Por primera vez, la FDA tendrá un mandato legislativo para exigir controles preventivos integrales y basados en la ciencia en toda la gama del suministro de alimentos. Este mandato incluye lo siguiente:

- Controles de prevención obligatorios para los establecimientos de alimentos: los establecimientos de alimentos deben aplicar un plan escrito de controles preventivos. Esto conlleva: (1) evaluar los peligros que podrían afectar a la inocuidad de los alimentos; (2) especificar los pasos o controles preventivos que se pondrán en práctica para minimizar o prevenir en gran parte los peligros; (3) especificar la manera en que el establecimiento vigilará estos controles para garantizar que den resultado; (4) mantener registros de rutina del monitoreo; y (5) especificar qué medidas tomará el establecimiento para corregir los problemas que surjan. (La norma definitiva está prevista para 18 meses después de la promulgación.)

- Normas obligatorias relacionadas con la inocuidad de los alimentos: la FDA debe establecer mínimas normas basadas en la ciencia para la producción y cosecha 
de frutas y verduras inocuas. Esas normas deben considerar los peligros de origen natural, así como los que se introduzcan inintencionada 0 intencionadamente, y deben abordar las enmiendas relacionadas con el suelo (los materiales agregados al suelo tales como el abono orgánico), la higiene, el envasado, los controles de temperatura, los animales en la zona de cultivo y el agua. (El reglamento definitivo está previsto para unos 2 años después de la promulgación.)

- Facultad de prevenir la contaminación intencional: la FDA debe emitir reglamentos para prevenir la adulteración intencional de alimentos, incluido el establecimiento de estrategias de mitigación basadas en la ciencia para preparar y proteger la cadena de suministro de alimentos en puntos vulnerables específicos. (La norma definitiva está prevista para 18 meses después de la promulgación.)

\section{Inspección y cumplimiento}

La FSMA reconoce que las normas de control preventivo mejoran la inocuidad de los alimentos sólo en la medida que los productores y procesadores las cumplan. Por lo tanto, será necesario que la FDA se encargue de la supervisión, garantice el cumplimiento de los requisitos y responda eficazmente cuando surjan problemas. La FSM A otorga a la FDA nuevos instrumentos importantes para la inspección y el cumplimiento, entre ellos:
- Frecuencia de la inspección obligatoria: la FSMA establece una frecuencia de inspección obligatoria, basada en el riesgo, de los establecimientos de alimentos y exige que la frecuencia de la inspección aumente inmediatamente. Todos los establecimientos nacionales de alto riesgo deben inspeccionarse dentro de los cinco años de la promulgación y no menos de cada tres años a partir de entonces. En el plazo de un año de la promulgación, la ley dispone que la FDA inspeccione como mínimo 600 establecimientos extranjeros y duplique esas inspecciones cada año durante los siguientes cinco años.

- Acceso a los registros: la FDA tendrá acceso a registros, incluidos planes de inocuidad de los alimentos de la industria, y las empresas de registros deberán seguir documentando la aplicación de sus planes.

- Pruebas por laboratorios acreditados: la FSMA exige que ciertas pruebas de alimentos sean efectuadas por laboratorios acreditados y ordena a la FDA establecer un programa de acreditación de laboratorios para garantizar que los laboratorios de prueba de alimentos de los Estados Unidos cumplan normas de alta calidad. (El establecimiento del programa de acreditación está previsto para 2 años después de la promulgación.) 


\section{Respuesta}

La FSMA reconoce que la FDA debe contar con los instrumentos necesarios para responder con eficacia cuando surgen problemas a pesar de los controles preventivos empleados. Algunas facultades nuevas son:

- Retirada obligatoria: la FSMA otorga a la FDA la facultad de disponer una retirada obligatoria cuando una empresa no retira voluntariamente alimentos poco seguros después de que dicho organismo se lo solicita.

- Ampliación de la detención administrativa: la FSM A otorga a la FDA una norma más flexible para la detención administrativa de productos que potencialmente infrinjan la ley (la detención administrativa es el procedimiento que emplea la FDA para prevenir el traslado de alimentos de dudoso origen).

- Suspensión del registro: la FDA puede suspender el registro de un establecimiento si determina que el alimento presenta una probabilidad razonable de graves consecuencias adversas para la salud o incluso la muerte. Un establecimiento suspendido tiene prohibido distribuir alimentos. (En vigor 6 meses después de la promulgación.)

- Mejor capacidad de rastreo de productos: la FDA debe establecer un sistema que mejore su capacidad de rastrear alimentos nacionales e importados y darles seguimiento. Además, la FDA debe establecer proyectos experimentales para investigar y evaluar métodos con el fin de identificar rápida y eficazmente los destinatarios de alimentos para prevenir o controlar el brote de una enfermedad transmitida por los alimentos. (La puesta en práctica de los proyectos experimentales está prevista para 9 meses después de la promulgación.)

- Mantenimiento adicional de registros para alimentos de alto riesgo: la FDA debe dictar la normalización propuesta con el fin de establecer requisitos de mantenimiento de registros para establecimientos que fabrican, elaboran, envasan 0 conservan alimentos que el Secretario designe como alimentos de alto riesgo. (Puesta en práctica prevista para 2 años después de la promulgación.)

\section{Importaciones}

La FSMA otorga a la FDA facultades sin precedentes para garantizar que los alimentos importados cumplan las normas de los EE.UU., y sean inocuos para los consumidores estadounidenses. Algunas facultades nuevas son:

- Responsabilidad de los importadores: por primera vez, los importadores tienen la responsabilidad explícita de verificar que sus proveedores extranjeros tengan los controles preventivos correspondientes para garantizar que el alimento que producen sea inocuo. (El reglamento y orientación definitivos están previstos para 1 año después de la promulgación.) 
- Certificación de terceros: la FSMA establece un programa por el cual terceros calificados pueden certificar que los establecimientos de alimentos extranjeros cumplen las normas estadounidenses de inocuidad de los alimentos. Esta certificación puede emplearse para facilitar el ingreso de las importaciones. (El establecimiento de un sistema para que la FDA reconozca a los organismos de acreditación está previsto para 2 años después de la promulgación.)

\section{"Un gobierno extranjero puede actuar en calidad de auditor externo"}

Las cooperativas y agencias gubernamentales extranjeras pueden recibir la acreditación para actuar en calidad de auditores externos.

- Certificación de alimentos de alto riesgo: la FDA tiene la facultad de exigir que los alimentos importados de alto riesgo vayan acompañados de una certificación creíble de terceros u otra garantía de cumplimiento como condición de ingreso en los EE. UU.

- Programa voluntario para importadores calificados: la FDA debe establecer un programa voluntario para importadores que disponga la agilización del examen e ingreso de alimentos procedentes de los importadores participantes. Entre otras cosas, la elegibilidad se limita a los importadores que ofrezcan alimentos de establecimientos certificados. (La puesta en práctica está prevista para 18 meses después de la promulgación.)
- Facultad de denegar el ingreso: la FDA podrá denegar el ingreso a los EE.UU., a los alimentos procedentes de un establecimiento extranjero si dicho establecimiento, o el país donde se encuentre dicho establecimiento, le niega acceso a la FDA.

\section{Mayor colaboración entre los organismos}

La FSMA establece un sistema formal de colaboración con otros organismos gubernamentales, tanto nacionales como extranjeros. Al hacerlo, la ley reconoce explícitamente que todos los organismos dedicados a la inocuidad de los alimentos deben colaborar juntos en forma integrada para lograr nuestras metas en materia de salud pública. Los siguientes son ejemplos de una mayor colaboración:

- Fomento de la capacidad estatal y local: la FDA debe formular y aplicar estrategias para aprovechar y mejorar la inocuidad de los alimentos y la capacidad de defensa de los organismos estatales y locales. La FSMA otorga a la FDA un mecanismo de subsidios plurianuales para facilitar la inversión en la capacidad estatal con el fin de lograr con mayor eficiencia las metas nacionales en materia de inocuidad de los alimentos.

- Fomento de la capacidad extranjera: la ley ordena a la FDA formular un plan integral para ampliar la capacidad de los gobiernos extranjeros y sus industrias. Un componente del plan es abordar la capacitación de los gobiernos extranjeros y productores de alimentos en cuanto a los 
requisitos de inocuidad de los alimentos de los EE. UU.

- Dependencia de las inspecciones que realicen otros organismos: la FDA tiene la facultad explícita de depender de las inspecciones que realicen otros organismos federales, estatales y locales para cumplir el mandato de mayor inspección de establecimientos nacionales. La FSMA también permite a la FDA celebrar acuerdos interinstitucionales para hacer uso de los recursos relativos a la inspección de establecimientos de mariscos, tanto nacionales como extranjeros, así como de importaciones de mariscos.

\section{Aranceles}

La FSMA autoriza a la FDA a cobrar aranceles por la segunda inspección de ciertos establecimientos de alimentos nacionales, establecimientos de alimentos extranjeros e importadores. No se cobra ningún arancel por la primera inspección de la FDA. El arancel de segunda inspección se cobra para costear los gastos relacionados con la segunda inspección cuando una inspección inicial identifica determinados problemas con la seguridad de los alimentos.

La FDA tiene autoridad para cobrar aranceles por actividades de revisión de alimentos relacionadas con un pedido de revisión cuando un establecimiento de alimentos nacional o un importador no cumplen con dicho pedido.

\section{Otras disposiciones claves sobre la normatividad}

Otorga más flexibilidad a la FDA para que ejerza su autoridad de detención administrativa con el fin de evitar que ingresen al mercado productos posiblemente adulterados o mal rotulados.

Las empresas deben continuar cumpliendo con las Buenas Prácticas de Manufactura actuales (CurrentGoodManufacturing Practices CGM P) y HACCP. Asimismo, la FDA no prevé que los nuevos requisitos de control preventivo reemplacen a las CGMP.

La FSMA prevé exenciones o requisitos modificados en ciertas circunstancias, por ejemplo, si un establecimiento ya tiene la obligación de cumplir o cumple con el HACCP para mariscos o jugos, o si un establecimiento es muy pequeño. La FDA se referirá a estos aspectos de la ley y los explicará en el marco del proceso normativo para implementar la disposición de controles preventivos. Se expedirán pautas, propuestas de reglas y reglas definitivas con suficiente antelación para que el público pueda emitir sus comentarios.

Para más información sobre las nuevas normas propuestas, puede ir a los siguientes enlaces:

Federal RegisterNoticefortheProposed Rule "CurrentGoodManufacturingPractice and HazardAnalysis and RiskBasedPreventiveControlsfor Human Food" 
http://www.ofr.gov/(X(1)S(v3yf3je4uhgifjgf t2sscuim))/OFRUpload/OFRData/201300125_PI.pdf

Federal RegisterNoticefortheProposed Rule "StandardsfortheGrowing, Harvesting, Packing and Holding of Produce for Human Consumption"

http://www.ofr.gov/(X(1)S(v3yf3je4uhgifjgf t2sscuim)//OFRUpload/OFRData/201300123 PI.pdf

La FDA insta a la industria en el exterior, a los gobiernos extranjeros, al público y otros miembros de la comunidad internacional a revisar y comentar sobre estas importantes propuestas. Las propuestas estarán abiertas para comentario del público durante los próximos 120 días. $^{3}$

La FDA también notificará al Comité de Medidas Sanitarias y Fitosanitarias (MSF) de la Organización Mundial del Comercio (OMC) sobre las reglas propuestas y las instrucciones del expediente ("docket") el mismo día que se publiquen en el Federal Register, programado tentativamente para el 7 de Enero del 2013. La publicación de la OMC aparecerá una semana después.

\section{CUADRO RESUMEN INFORMATIVO}

Las variables incluidas en este cuadro informativo son:

Consignatario: Se refiere al nombre del establecimiento declarado que es responsable del producto rechazado.

Dirección del Consignatario: Se refiere a la dirección del establecimiento en el país de origen.
Código del Producto: Es un identificador único asignado al producto regulado por la FDA $^{4}$.

Descripción del Producto Rechazado: Es la descripción del producto ofrecido por el importador a la entrada de las aduanas.

Código de Violación de la FDA: Identifica la violación para que la FDA ejecute la detención.

Razón de Rechazo: Describe la razón específica de la detención ${ }^{5}$. 


\begin{tabular}{|c|c|c|c|c|c|c|c|}
\hline Conigntario & $\begin{array}{l}\text { Drecióndd } \\
\text { conigigtario } \\
\text { (duded/pás) }\end{array}$ & $\begin{array}{l}\text { Colgoda } \\
\text { producto }\end{array}$ & $\begin{array}{l}\text { Pescripiónd: } \\
\text { produdo } \\
\text { rederadb }\end{array}$ & \begin{tabular}{c|} 
đodgode \\
vidadónde \\
laFDA
\end{tabular} & $\begin{array}{l}\text { Reánce } \\
\text { redero }\end{array}$ & $\begin{array}{l}\text { Fedhace } \\
\text { redero }\end{array}$ & $\begin{array}{l}\text { (Dstrito } \\
\text { reqponsaddedd } \\
\text { redead) }\end{array}$ \\
\hline $\begin{array}{l}\text { Pitll } \\
\text { laboratories }\end{array}$ & $\begin{array}{c}\text { Ca.Sury10a } \\
\text { COe \#400 }\end{array}$ & 68А999 & $\begin{array}{l}\text { SDAGRP } \\
\text { ANIGRALID } \\
\text { capules }\end{array}$ & $\begin{array}{l}\text { NOTISIED } \\
\text { UAFROEO }\end{array}$ & $\begin{array}{l}\text { EIQEADO } \\
\text { NOESA } \\
\text { ARCBAD }\end{array}$ & 26Doc2012 & $\begin{array}{l}\text { PADO(Nitiand } \\
\text { Forick) }\end{array}$ \\
\hline $\begin{array}{l}\text { Núáizabeth } \\
\text { Atbiza }\end{array}$ & $\begin{array}{c}\text { Condonivio } \\
\text { Balnary Edficio } \\
\text { 4diMjicanos }\end{array}$ & 6.CD48 & $\begin{array}{c}\text { BACQ } \\
\text { ANIGRPA4FA } \\
\text { REG\# 11080880036 }\end{array}$ & $\begin{array}{l}\text { NOISIED } \\
\text { UAPROAD }\end{array}$ & $\begin{array}{c}\text { EIQEADO } \\
\text { NDEIA } \\
\text { ARCADO }\end{array}$ & 31-Dec2012 & $\begin{array}{c}\text { NADOVanaica } \\
\text { NewYark) }\end{array}$ \\
\hline $\begin{array}{l}\text { Núáizabeth } \\
\text { Atbiza }\end{array}$ & $\begin{array}{c}\text { Condonirio } \\
\text { Balnary Edficio } \\
\text { 4diNicanos }\end{array}$ & 60AD & $\begin{array}{l}\text { AKASBT/R/FDA } \\
\text { REG1T80988636 }\end{array}$ & $\begin{array}{l}\text { NOTISIED } \\
\text { UAPFOAD }\end{array}$ & $\begin{array}{c}\text { EIQEALO } \\
\text { NDESTA } \\
\text { ARCBAD }\end{array}$ & 31-Dec2012 & $\begin{array}{l}\text { NADOOlanaica } \\
\text { NewYork) }\end{array}$ \\
\hline $\begin{array}{l}\text { Mrría日izabeth } \\
\text { Atbiza }\end{array}$ & $\begin{array}{l}\text { Condonirio } \\
\text { Balnayy Edifico } \\
\text { 4diMjicanos }\end{array}$ & 6.0058 & 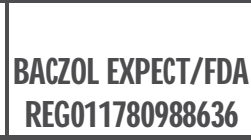 & $\begin{array}{l}\text { NOLISIED } \\
\text { UAFROAD }\end{array}$ & $\begin{array}{c}\text { EIQEADO } \\
\text { NDEIA } \\
\text { ARCBAD }\end{array}$ & B-Dec-2012 & $\begin{array}{l}\text { NAPOOlanaica } \\
\text { NewYork) }\end{array}$ \\
\hline $\begin{array}{l}\text { Neŕa日izabeth } \\
\text { Atbiza }\end{array}$ & $\begin{array}{l}\text { Condbríio } \\
\text { Balnaýrificio } \\
\text { 4diNicanos }\end{array}$ & 60NAT6 & $\begin{array}{r}\text { VRMEXFCA } \\
\text { REG\#11180988\%60 }\end{array}$ & $\begin{array}{l}\text { NOUSIED/ } \\
\text { UAFROEO }\end{array}$ & $\begin{array}{l}\text { EIQEIADO ND } \\
\text { EIAARCBDO }\end{array}$ & 2012 & $\begin{array}{c}\text { NMEDOVanaica } \\
\text { NewYark) }\end{array}$ \\
\hline $\begin{array}{l}\text { Nuŕa日izabeth } \\
\text { Atbiza }\end{array}$ & $\begin{array}{l}\text { Condbinio } \\
\text { BdhatyEodifio } \\
\text { 4di|icanos }\end{array}$ & 6aDAal & $\begin{array}{r}\text { PANAD/FDA } \\
\text { REG\#11000888606 }\end{array}$ & $\begin{array}{l}\text { NOTISIEY } \\
\text { UNAROAD }\end{array}$ & $\begin{array}{l}\text { EIQETADO ND } \\
\text { ESAARCBDD }\end{array}$ & 2012 & $\begin{array}{c}\text { NAPOVlanaica } \\
\text { NewYork) }\end{array}$ \\
\hline $\begin{array}{l}\text { Máabircheth } \\
\text { Atbiza }\end{array}$ & $\begin{array}{l}\text { Condbrinio } \\
\text { Balnaýtificio } \\
\text { 4diMicanos }\end{array}$ & 60DACI & 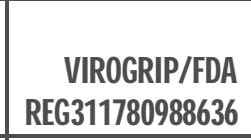 & $\begin{array}{l}\text { NOUSIED } \\
\text { UAPFOAD }\end{array}$ & $\begin{array}{l}\text { EIQEIADO ND } \\
\text { ESAARCBDO }\end{array}$ & 2012 & $\begin{array}{l}\text { NATOOlanaica } \\
\text { NewYork) }\end{array}$ \\
\hline $\begin{array}{l}\text { Núáizabeth } \\
\text { Atbiza } \\
\end{array}$ & $\begin{array}{l}\text { Condoninio } \\
\text { BalnayEdficio } \\
\text { 4dingicanos } \\
\end{array}$ & GADAOV & 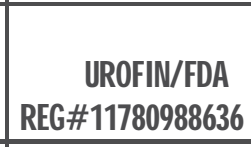 & $\begin{array}{l}\text { NOUSIED } \\
\text { UAFROA }\end{array}$ & $\begin{array}{l}\text { EIQEADO NO } \\
\text { BTAARCADD }\end{array}$ & 2012 & $\begin{array}{c}\text { MADOVanaica } \\
\text { NewYork) }\end{array}$ \\
\hline $\begin{array}{l}\text { Núáizabeth } \\
\text { Albiza }\end{array}$ & 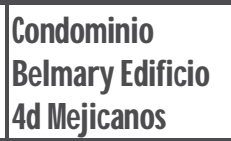 & 6apali. & $\begin{array}{c}\text { PANAM } \\
\text { EXIRAFDA } \\
\text { REG } 110098866\end{array}$ & $\begin{array}{l}\text { NOUSIED } \\
\text { UAFROAD }\end{array}$ & $\begin{array}{l}\text { EIQEACD ND } \\
\text { ETAARCEADO }\end{array}$ & 012 & $\begin{array}{l}\text { NADOV lanaica, } \\
\text { NewYork) }\end{array}$ \\
\hline $\begin{array}{l}\text { Máalizabeth } \\
\text { Albiza }\end{array}$ & $\begin{array}{l}\text { Condoninio } \\
\text { BdnaryEoficio } \\
\text { 4din:icanos }\end{array}$ & 6aDAOI & $\begin{array}{l}\text { DOGN/FA } \\
\text { REGH11780988606 }\end{array}$ & $\begin{array}{l}\text { NOTISIED } \\
\text { UAFROAD }\end{array}$ & $\begin{array}{l}\text { EIQFACD ND } \\
\text { ETAARCEADO }\end{array}$ & $=2012$ & $\begin{array}{c}\text { MADOVanaica } \\
\text { NewYork) }\end{array}$ \\
\hline $\begin{array}{l}\text { Mría日izabell } \\
\text { Atbiza }\end{array}$ & $\begin{array}{l}\text { Condbrinio } \\
\text { Balnay Edficio } \\
\text { 4din:icanos }\end{array}$ & 6aDAl & 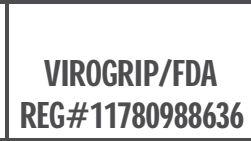 & $\begin{array}{l}\text { NOUISIEY } \\
\text { UAPFOAD }\end{array}$ & $\begin{array}{l}\text { EIQEIADO ND } \\
\text { ESAARCBAD }\end{array}$ & 31-Dec2012 & $\begin{array}{c}\text { NAEDOlanaica } \\
\text { NewYork) }\end{array}$ \\
\hline $\begin{array}{l}\text { Mríäizabel } \\
\text { Arbiza }\end{array}$ & $\begin{array}{l}\text { Condbinio } \\
\text { BalmaryEdficio } \\
\text { 4diMjicanos }\end{array}$ & 6aDACI & $\begin{array}{c}\text { URA } \\
\text { DUPEXFA } \\
\text { REC\#110898856 }\end{array}$ & $\begin{array}{l}\text { NOUSIED } \\
\text { UNFPOAD }\end{array}$ & $\begin{array}{l}\text { EIQEIADO ND } \\
\text { EIAARCADO }\end{array}$ & 2012 & $\begin{array}{c}\text { NAFOOlanaica } \\
\text { NewYark) }\end{array}$ \\
\hline $\begin{array}{l}\text { Máa日izabeth } \\
\text { Atbiza }\end{array}$ & $\begin{array}{l}\text { Condbninio } \\
\text { BalmaryEdfidio } \\
\text { 4dinjicanos }\end{array}$ & 6adar & $\begin{array}{c}\text { PANAD } \\
\text { EXIRAFA } \\
\text { REC\#11T8088\%6 }\end{array}$ & $\begin{array}{l}\text { NOLISIEY } \\
\text { UAPFOHD }\end{array}$ & $\begin{array}{l}\text { EIQEIADO ND } \\
\text { ETAARCBAD }\end{array}$ & 2012 & $\begin{array}{c}\text { NATDONanaica } \\
\text { NewYork) }\end{array}$ \\
\hline
\end{tabular}




\begin{tabular}{|c|c|c|c|c|c|c|c|}
\hline Consic & $\begin{array}{l}\text { Dreeciónda } \\
\text { consigntaio } \\
\text { (ductadpás) }\end{array}$ & $\begin{array}{l}\text { dgodla } \\
\text { odtato }\end{array}$ & $\begin{array}{l}\text { posoi } \\
\text { prodetc }\end{array}$ & $\begin{array}{c}\text { Cólgoded } \\
\text { vidaióndela } \\
\text { FAA }\end{array}$ & Pazónderectero & $\begin{array}{l}\text { Fertace } \\
\text { rechexo }\end{array}$ & $\begin{array}{l}\text { (Distito } \\
\text { responsaddeda } \\
\text { reatero) }\end{array}$ \\
\hline $\begin{array}{l}\text { nálactoth } \\
\text { beiza }\end{array}$ & $\begin{array}{l}\text { BdmaryEdifido } \\
\text { 4dimicanos }\end{array}$ & 6apral & 20 & $\begin{array}{c}\text { NOISIEY } \\
\text { UNAPFRO } \\
\text { VDD }\end{array}$ & $\begin{array}{l}\text { EIQEADOS NO } \\
\text { ETAARCBDO }\end{array}$ & and & $\begin{array}{c}\text { NKAD } \\
\text { (Jansica New } \\
\text { Yark) }\end{array}$ \\
\hline doth & $\begin{array}{l}\text { Candoninio } \\
\text { BalnaryÉfício } \\
\text { 4dingicanos }\end{array}$ & iDral & DA & $\begin{array}{l}\text { NOUSIED/ } \\
\text { UAFROAD }\end{array}$ & $\begin{array}{r}\text { EIQ } \\
\mathbf{N} \\
\mathbf{A P}\end{array}$ & 2012 & $\begin{array}{c}\text { NKAD } \\
\text { (Jantica New } \\
\text { Yark) }\end{array}$ \\
\hline $\begin{array}{l}\text { Mría日izdbeth } \\
\text { Atbeiza }\end{array}$ & $\begin{array}{l}\text { Condonirio } \\
\text { BdmaryEdficio } \\
\text { 4digicanos }\end{array}$ & 1 & $\begin{array}{l}\text { SUDA } \\
\text { REGIIT }\end{array}$ & $\begin{array}{l}\text { NOJISIE/ } \\
\text { UAFROAD }\end{array}$ & $\begin{array}{r}\mathbf{E I Q} \\
\mathbf{N} \\
\mathbf{A F}\end{array}$ & 2012 & $\begin{array}{c}\text { NATD } \\
\text { (Jantica, Naw } \\
\text { Yark) }\end{array}$ \\
\hline $\begin{array}{l}\text { ińa日izabeth } \\
\text { ibaiza }\end{array}$ & $\begin{array}{l}\text { Candoninio } \\
\text { BalmanyEdifio } \\
\text { 4digicanos }\end{array}$ & 08 & $\begin{array}{r}\text { TA } \\
\text { ADII } \\
\text { REAII }\end{array}$ & $\begin{array}{l}\text { NOJISIEY } \\
\text { UAFROAD }\end{array}$ & $\begin{array}{r}\text { EIC } \\
\mathbf{N} \\
\mathbf{A F}\end{array}$ & 2012 & $\begin{array}{c}\text { NATD } \\
\text { (Jantica, New } \\
\text { Yark) }\end{array}$ \\
\hline $\begin{array}{l}\text { Ińa日izabeth } \\
\text { teiza }\end{array}$ & $\begin{array}{l}\text { Candonirio } \\
\text { BdmaryEdicio } \\
\text { 4digicanos }\end{array}$ & $\mathbf{B}$ & $\begin{array}{r}\text { TA } \\
\text { INFAN } \\
\text { EGHIT }\end{array}$ & $\begin{array}{l}\text { NOJISIEY } \\
\text { UAFROAD }\end{array}$ & $\begin{array}{r}\text { ElQ } \\
\mathbf{N} \\
\mathbf{A P} \\
\end{array}$ & 012 & $\begin{array}{c}\text { NATD } \\
\text { (Jantica Naw } \\
\text { Yark) }\end{array}$ \\
\hline $\begin{array}{l}\text { ńa日iz } \\
\text { beiza }\end{array}$ & $\begin{array}{l}\text { Candani } \\
\text { Balnary } \\
\text { 4dingic }\end{array}$ & al & $\begin{array}{r}\text { VRO } \\
\text { EG\#II } \\
\end{array}$ & $\begin{array}{l}\text { NOUSIEY } \\
\text { UAFROAD }\end{array}$ & $\begin{array}{l}\bar{D} \\
\mathbf{D}\end{array}$ & 2012 & $\begin{array}{c}\text { NसAD } \\
\text { (Jantica Naw } \\
\text { Yark) }\end{array}$ \\
\hline $\begin{array}{l}\text { Iría日izdoth } \\
\text { toiza }\end{array}$ & $\begin{array}{l}\text { Candonirio } \\
\text { BdmaryEdifio } \\
\text { 4dígicanos }\end{array}$ & & $\begin{array}{l}\text { VRC } \\
\text { ECNTE }\end{array}$ & $\begin{array}{l}\text { NOUSIED/ } \\
\text { UAFROAD }\end{array}$ & $\begin{array}{r}\text { EIC } \\
1 \\
\mathbf{A F}\end{array}$ & 012 & $\begin{array}{c}\text { NATD } \\
\text { (Jantica New } \\
\text { Yark) }\end{array}$ \\
\hline $\begin{array}{l}\text { hía日izcheth } \\
\text { haiza }\end{array}$ & $\begin{array}{l}\text { Candar } \\
\text { Bainery } \\
\text { 4dingic }\end{array}$ & & $\begin{array}{l}\text { SDA } \\
\text { EG\#1D }\end{array}$ & $\begin{array}{l}\text { NOISIED } \\
\text { UAFROED }\end{array}$ & $\bar{D}$ & 2012 & $\begin{array}{c}\text { NKAD } \\
\text { (Jantica, New } \\
\text { Yark) }\end{array}$ \\
\hline $\begin{array}{l}\text { lría日|zdoeth } \\
\text { taiza }\end{array}$ & $\begin{array}{l}\text { Condoninio } \\
\text { BalnaryEofício } \\
\text { 4digicanos }\end{array}$ & & $\begin{array}{l}\text { TABĆVC } \\
\text { REGAII }\end{array}$ & $\begin{array}{l}\text { NOUSIED } \\
\text { UAFROED }\end{array}$ & $\begin{array}{r}\text { EIC } \\
\text { AP } \\
\end{array}$ & 2012 & $\begin{array}{c}\text { NKAD } \\
\text { (Jantica New } \\
\text { Yark) }\end{array}$ \\
\hline $\begin{array}{l}\text { MrńaEirabeth } \\
\text { Arleiza }\end{array}$ & $\begin{array}{l}\text { Condaninio } \\
\text { Bdmay } \\
\text { Edfidb4d }\end{array}$ & & DEOA & $\begin{array}{l}\text { TUSIED } \\
\text { ATROAD }\end{array}$ & $\begin{array}{l}\mathbf{D I} \\
\mathrm{A} \\
\mathrm{D}\end{array}$ & 2012 & $\begin{array}{c}\text { NATD } \\
\text { (Jantica New } \\
\text { York) }\end{array}$ \\
\hline $\begin{array}{l}\text { Mrríablzabeth } \\
\text { Albaiza }\end{array}$ & $\begin{array}{l}\text { Candonirio } \\
\text { BalnaryEdifio } \\
\text { 4digicanos }\end{array}$ & & BPASND & $\begin{array}{l}\text { NOUSID } \\
\text { UAFROED }\end{array}$ & $\begin{array}{r}\text { ElQ } \\
\text { NO } \\
\text { AR }\end{array}$ & 2012 & $\begin{array}{c}\text { NATD } \\
\text { (Jantica New } \\
\text { Yark) }\end{array}$ \\
\hline $\begin{array}{l}\text { Mría日izabeth } \\
\text { Arbaiza }\end{array}$ & $\begin{array}{l}\text { Condoninio } \\
\text { Bdmay } \\
\text { Edfídb4d }\end{array}$ & & $\begin{array}{l}\text { NEOFNC } \\
\text { ARECWITK }\end{array}$ & $\begin{array}{l}\text { NOISIED } \\
\text { UAFROAD }\end{array}$ & $\begin{array}{r}\text { EIQ } \\
\mathbf{N} \\
\mathbf{A F}\end{array}$ & 2012 & $\begin{array}{c}\text { NKAD } \\
\text { (Jantica New } \\
\text { York) }\end{array}$ \\
\hline Mría日irabelh & $\begin{array}{l}\text { Candonirio } \\
\text { Bantry } \\
\text { Edfídb4d }\end{array}$ & & $\begin{array}{l}\text { DOAFN/FA } \\
\text { EG\#1180988666 }\end{array}$ & $\begin{array}{l}\text { NOUSIED } \\
\text { UNAROBD }\end{array}$ & $\begin{array}{c}\text { EIQEIADY } \\
\text { NDEIA } \\
\text { ARCALO }\end{array}$ & 2012 & $\begin{array}{c}\text { NADD } \\
\text { (Jantica New } \\
\text { York) }\end{array}$ \\
\hline $\begin{array}{l}\text { Mrta日i } \\
\text { Arbeiza }\end{array}$ & $\begin{array}{l}\text { Condoninio } \\
\text { BalnaryEdifio } \\
\text { 4díicanos }\end{array}$ & J & URC & $\begin{array}{l}\text { NOUSIED } \\
\text { UNAROED }\end{array}$ & $\begin{array}{r}\text { EIQF } \\
\text { NOE } \\
\text { ARQ }\end{array}$ & 2012 & $\begin{array}{c}\text { NKAD } \\
\text { (Jantica, New } \\
\text { Yark) }\end{array}$ \\
\hline
\end{tabular}

Fuente: Creación propia, basado en datos OASIS/ FDA 
Fecha de Rechazo:_Identifica la fecha de cuando la acción fue realizada.

Distrito Responsable del Rechazo: Identifica la oficina del distrito de la FDA en donde tiene jurisdicción el producto rechazado, es decir, el puerto por donde entra el producto.

\section{CONCLUSIONES}

El aumento de los controles en la inocuidad de los alimentos al mercado de los Estados Unidos es una realidad con esta Ley, por lo tanto, lo más sensato es que los países que tienen relaciones comerciales con los EE.UU., traten de anticiparse y actuar proactivamente en implementar acciones concretas, para que a corto y mediano plazo se puedan homologar los sistemas de control en la inocuidad de los alimentos entre ambas naciones.

En el cuadro del resumen informativo, podemos apreciar todos los rechazos de los productos salvadoreños que al llegar a las aduanas de los EEUU fueron detenidos por la FDA, para los meses de noviembre y diciembre del 2012, sin embargo, se puede distinguir que para el mes de noviembre no hubo ningún rechazo, únicamente para el mes de diciembre, en el cual arroja un dato de 27 rechazos.

Del número de rechazos anteriormente descritos, se debe prestar atención que fueron ocasionados únicamente por 2 empresas, una de ellas solo tuvo un rechazo y la otra fue objeto de 26 productos rechazados, ambas coinciden en las causales de los rechazos, por lo tanto cada producto detenido tuvo 2 razones objeto de rechazo. Hay que tomar en cuenta que al totalizar el número de rechazos, el resultado se doblara en forma general, ya que la FDA contabiliza por cada razón de rechazo ${ }^{6}$.

De los 27 productos rechazados en el mes de diciembre, la FDA no realizó ningún análisis a las muestras de los productos, ya que a simple vista pudieron detectar el cargo de violación a la Ley. Cabe destacar, que ninguno de estos productos rechazados presenta en los registros de la FDA, antecedentes de recibo de análisis de laboratorios privados.

A continuación se muestra el desempeño de las causales de rechazos en los meses de noviembre-diciembre 2012:

\begin{tabular}{c|c} 
& DESCRIPCIÓN CÓDIGO INDUSTRIAL \\
\hline 66 & Medicinas para las personas y los animales \\
\hline 61 & Medicinas para las personas y los animales \\
\hline 60 & Medicinas para las personas y los animales \\
\hline 64 & Medicinas para las personas y los animales \\
\hline 56 & Antibióticos (humano/animal) \\
\hline
\end{tabular}

Fuente: Creación propia basado en datos OASIS/FDA

De manera general se puede observar que el mal etiquetado representa un $50 \%$ y la droga o fármaco no aprobado el otro $50 \%$ El número real de los productos rechazados fueron de 27 envíos y el cargo por violaciones realizadas fueron 54 .

Cabe comentar que el riesgo implícito en estas violaciones es muy variable, sin embargo, cuando se trata de fármacos para la FDA es de mucho riesgo. El hecho de que un medicamento no tenga toda la 
DESCRIPCIÓN DE LA RAZON DE RECHAZO

\begin{tabular}{l|l}
\hline & Razón: NO ESTA LISTADO \\
Sección 502(0), 801(a)(3); MAL \\
ETIQUETADO. Cargo: Parece \\
que al fármaco o el dispositivo \\
no esta incluido en una lista \\
requerida por la sección 510(j), \\
o un anuncio u otro tipo de \\
información al respecto no fue \\
proporcionada según lo \\
requerido por la sección 510(j) \\
0 510(k)
\end{tabular}

Fuente: creación propia, basado en datos OAIS/FDA información requerida según los requerimientos de la FDA en la etiqueta (NOT LISTED) es igual de peligrosa que si dejaran entrar un fármaco que no esté aprobado, porque no ha llenado la hoja registro requerida y haber pasado los controles de seguridad para la salud (UNAPPROVED).

Todas estas exportaciones realizadas, se llevaron a cabo de una manera formal. De acuerdo a entrevista con personal de la FDA, las detenciones que son consideradas informales, es decir, personas que llevan productos en sus maletas cuando viajan o los envíos que hacen a través de correo, no son reportadas por la FDA, ya que quien las realiza es US Customs and Border Protection.

Se debe ir detectando cuales son las debilidades que va presentando la industria de alimentos y bebidas en El Salvador, para lo cual se requerirá ir monitoreando cada mes si surgen otras razones de rechazo que ameriten ser tomadas en cuenta para implementar una medida más directa a las empresas respecto a necesidades de capacitación y así, poderles brindar una respuesta oportuna.

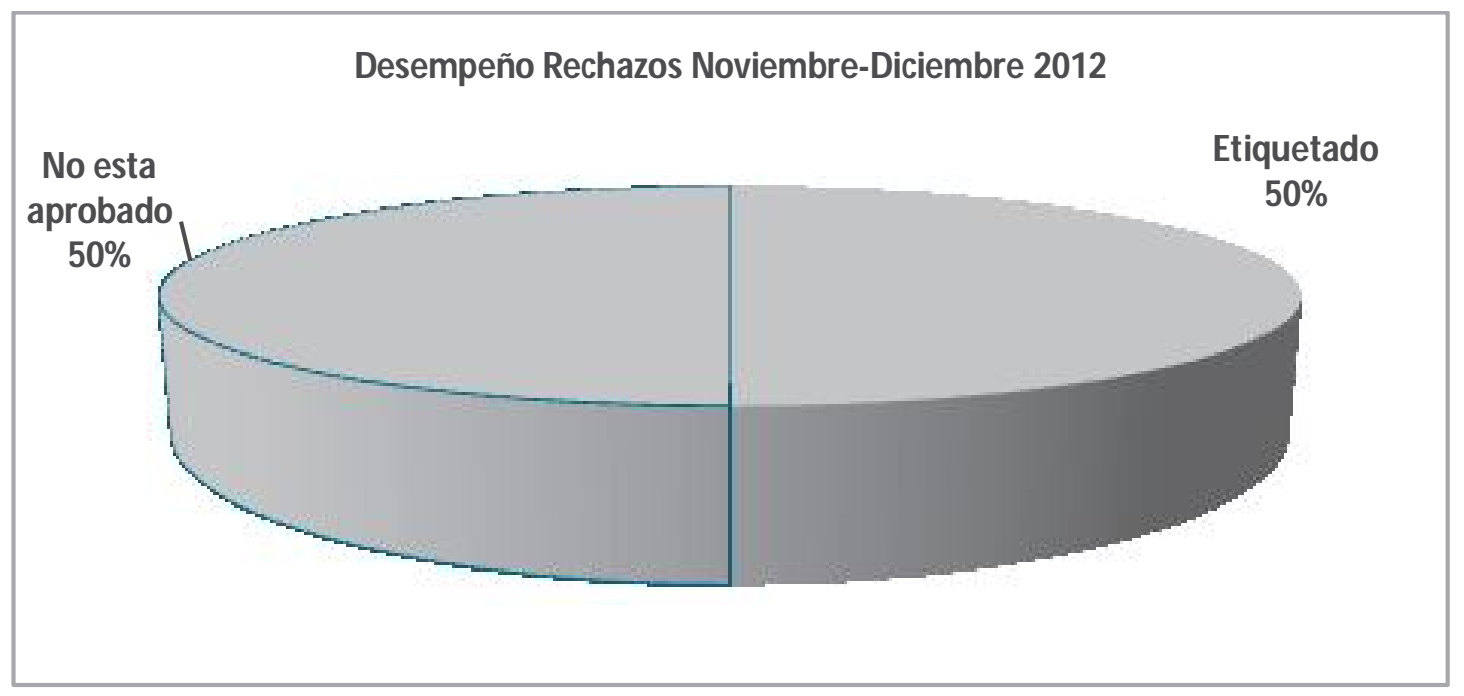

Fuente: Creación propia, basados en datos de Cuadro Informativo 


\section{RECOMENDACIONES}

Divulgar la nueva Ley de Inocuidad de alimentos a través de capacitaciones orientadas al sector empresarial cuando ya esté aprobada en el Federal Register.

Implementar un programa de Verificación de Proveedores del sector alimenticio, para que se pueda documentar debidamente la trazabilidad de este eslabón dentro de la cadena productiva alimenticia.

Solicitar al gobierno de los EEUU, que una entidad gubernamental Salvadoreña, pueda recibir la acreditación para actuar en calidad de auditor externo.

Llevar a cabo un Programa Voluntario de Exportadores Calificados que exige el gobierno de los EEUU para agilizar los envíos y evitar demoras en las aduanas.

Establecer un sistema integrado a nivel nacional de inocuidad de los alimentos en asociación con las instituciones públicas, académicas y privadas, en colaboración con el gobierno de los EE.UU.

Promover la puesta en práctica de una estrategia nacional de defensa agroindustrial.

Establecer un consorcio integrado de redes de laboratorios a nivel nacional para asegurar la calidad de sus servicios.

Mejorar la vigilancia de enfermedades transmitidas por los alimentos a nivel nacional, a través de instituciones públicas y privadas.

Capacitar a funcionarios del gobierno, de los diferentes departamentos del país. A continuación involucrados con el quehacer exportador, en la inocuidad de alimentos.

\section{Notas y referencias}

${ }^{1}$ Por sus siglas en ingles

${ }^{2}$ Fuente: FDA

${ }^{3}$ Fuente: Entrevista FDA

${ }^{4}$ Ver Descripción Código Industrial.

${ }^{5}$ Ver Descripción de la Razón de Rechazo.

${ }^{6}$ Ver figura desempeño rechazos noviembre-diciembre 2012

\section{Bibliografía}

- Allshouse, J, J. Buzby, D. Harvey, and D. Zorn. " International Trade and Food Safety: Economic Theory and Case Studies, AER-828, Chapter 7, U.S. Department of Agriculture, Economic Research Service, Nov. 2003.

- Code of Federal Regulations. Title 21 on Food and Drugs, Chapter 1, April 1, 2006 revision, Food and Drug Administration, Department of Health and Human Services.

- Calvin, L. "Produce, Food Safety, and International Trade: Response toU.S. Foodborne Illness Outbreaks Associated with Imported Produce,"International Trade and Food Safety: Economic Theory and Case Studies,AER-828, Chapter 5, U.S. Department of Agriculture, Economic ResearchService, Nov. 2003.

- Josling, Tim, Donna Roberts and David Orden (2004), Food Regulation and Trade. Toward a safe and open global system, Washington D.C., Institute for International Economics.

\section{Recursos Web}

- http://www.fda.gov/

- www.accessdata.fda.gov/scripts/cdrh/cfdo $\mathrm{cs} / \mathrm{cfcfr} / \mathrm{cfrsearch} . \mathrm{cfm}$.

- $\quad$ www.ers.usda.gov/publications/aer828/. 